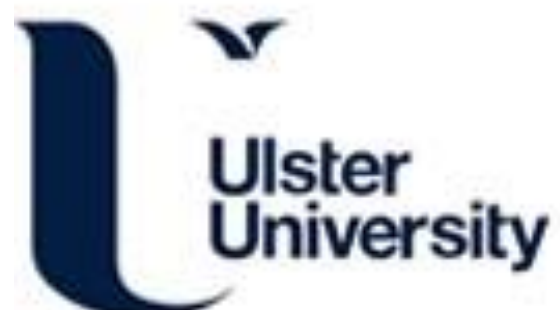

\section{Emulsion Electrospinning of Polytetrafluoroethylene (PTFE) Nanofibrous Membranes for High-Performance Triboelectric Nanogenerators}

Zhao, P., Soin, N., Prashanthi, K., Chen, J., Dong, S., Zhou, E., Zhu, Z., Narasimulu, A. A., Montemagno, C. D., Yu, L., \& Luo, J. (2018). Emulsion Electrospinning of Polytetrafluoroethylene (PTFE) Nanofibrous Membranes for High-Performance Triboelectric Nanogenerators. ACS Applied Materials and Interfaces, 10(6), 5880-5891. https://doi.org/10.1021/acsami.7b18442

Link to publication record in Ulster University Research Portal

Published in:

ACS Applied Materials and Interfaces

Publication Status:

Published (in print/issue): 14/02/2018

DOI:

10.1021/acsami.7b18442

Document Version

Author Accepted version

\section{General rights}

Copyright for the publications made accessible via Ulster University's Research Portal is retained by the author(s) and / or other copyright owners and it is a condition of accessing these publications that users recognise and abide by the legal requirements associated with these rights.

\section{Take down policy}

The Research Portal is Ulster University's institutional repository that provides access to Ulster's research outputs. Every effort has been made to ensure that content in the Research Portal does not infringe any person's rights, or applicable UK laws. If you discover content in the Research Portal that you believe breaches copyright or violates any law, please contact pure-support@ulster.ac.uk. 


\section{Emulsion electrospinning of polytetrafluoroethylene (PTFE) nanofibrous membranes for high-performance triboelectric nanogenerators}

Pengfei Zhao ${ }^{1}$, Navneet Soin ${ }^{1}$, Kovur Prashanthi ${ }^{2}$, Jinkai Chen ${ }^{3}$, Shurong Dong ${ }^{3}$, Erping Zhou $^{1}$, Zhigang Zhu ${ }^{4}$, Anand Arcot Narasimulu ${ }^{1}$, Carlo D Montemagno ${ }^{5}$, Liyang $\mathrm{Yu}^{3,}$, and Jack (Jikui) Luo ${ }^{1,3, *}$

${ }^{1}$ Institute for Materials Research \& Innovation (IMRI), School of Engineering, University of Bolton, Deane Road, Bolton, BL3 5AB, United Kingdom

${ }^{2}$ Ingenuity Lab, Department of Chemical and Materials Engineering, University of Alberta, Edmonton, Alberta, T6G 2V4, Canada

${ }^{3}$ Key Laboratory of RF Circuit and System, Ministry of Education, Hangzhou Dianzi University, Hangzhou 310018, China

${ }^{4}$ School of Environmental and Materials Engineering, College of Engineering, Shanghai Polytechnic University, Shanghai 201209, P. R. China

${ }^{5}$ Southern Illinois University, Carbondale, Illinois 62901, United States of America

\section{*Corresponding Author:}

Dr Liyang Yu,

Email: yuliyang@hdu.edu.cn

Prof. Jikui Luo,

Email: j.luo@bolton.ac.uk

Keywords. Triboelectric nanogenerator; Emulsion electrospinning; PTFE nanofiber; PEO carrier; Ion-injection; Electrostatic Force Microscopy (EFM). 


\begin{abstract}
Electrospinning is a simple, versatile technique for fabricating fibrous nanomaterials with the desirable features of extremely high porosities and large surface areas. Using emulsion electrospinning, polytetrafluoroethylene/polyethene oxide (PTFE/PEO) membranes were fabricated followed by a sintering process to obtain pure PTFE fibrous membranes, which were further utilised against a polyamide 6 (PA6) membrane for vertical contact-mode triboelectric nanogenerators (TENGs). Electrostatic force microscopy (EFM) measurements of the sintered electrospun PTFE membranes revealed the presence of both positive and negative surface charges owing to the transfer of positive charge from PEO which further corroborated by FTIR measurements. To enhance the ensuing triboelectric surface charge, a facile negative charge-injection process was carried out onto the electrospun (ES) PTFE subsequently. The fabricated TENG gave a stabilised peak-to-peak open-circuit-voltage $\left(\mathrm{V}_{\text {oc }}\right)$ of up to $\sim 900 \mathrm{~V}$, a short-circuit current density $\left(\mathrm{J}_{\mathrm{sc}}\right)$ of $\sim 22 \mathrm{mAm}^{-2}$ and a corresponding charge density of $\sim 149 \mu_{\mathrm{Cm}^{-2}}$, which are $\sim 12,14$ and 11 times higher than the corresponding values prior to the ion-injection treatment. This increase in surface charge density is caused by the inversion of positive surface charges with the simultaneous increase in the negative surface charge on the PTFE surface, which was confirmed by using EFM measurements. The negative charge injection led to an enhanced power output density of $\sim 9 \mathrm{Wm}^{-2}$ with high stability as confirmed from the continuous operation of the ion-injected PTFE/PA6 TENG for 30,000 operation cycles, without any visible reduction in the output. The work thus introduces a relatively simple, cost-effective and environmentally friendly technique for fabricating fibrous fluoropolymer polymer membranes with high thermal/chemical resistance in TENG field and an ion-injection method which is able to dramatically improve the surface charge density of the PTFE fibrous membranes.
\end{abstract}




\section{Introduction}

The development of nanogenerators as a type of physical devices to convert abundant waste mechanical energy into electricity has gathered significant pace over the last decade or so. Especially, the rapid development of portable devices and demands for battery-free, micro/nano- self-powered sensor systems is pushing new developments in the field of energy harvesting. Triboelectric nanogenerators (TENG), first developed by Prof. Wang's group in $2012^{1}$, have scaled up quite rapidly in terms of their power output with current reported values of hundreds of watts per square meters. ${ }^{2}$ In fact, TENG based self-powered systems were demonstrated to effectively harvest mechanical energy from human motions, such as vibration during walking, ${ }^{3}$ finger typing, ${ }^{4}$ and from ambient sources such as water waves ${ }^{5-7}$ or even the wind. ${ }^{8,9}$ The operation of TENGs is based on a judicious use of materials, with the highest power output from material combinations which are further apart in the so-called triboelectric series. $^{10}$

Polytetrafluoroethylene (PTFE), as one of the most triboelectric negative materials listed in the series, has been widely utilised in the area of TENGs for both energy harvesting and sensing devices. ${ }^{4,6,7,11,12}$ PTFE has a helical chain confirmation caused by the strong repellence amongst the fluorine atoms, thus providing the carbon backbone chain with a uniform coverage of fluorine which makes it highly inert to nearly all chemicals. Due to its high chemical resistance and thermal stability, ${ }^{13,14}$ most of the reported research on the use of PTFE for TENGs has focused on the use of commercial thin-film PTFE without any surface treatment ${ }^{4}$ or the use of treated PTFE films by expensive techniques like reactive ion etching $^{15,16}$ for forming micro/nanostructures on the surfaces. It is commonly recognised that the enhancement of the surface areas by micro/nanostructures enhances the effective surface charge density, hence the power output of the TENG during the energy harvesting (contact/friction) process. ${ }^{17-20}$ Zhang et al. ${ }^{21}$ reported on nanowire structures on the surface of the PTFE thin-films by applying inductively coupled plasma reactive ion etching (ICP RIE) technology with a thin gold mask. Similarly, using a copper etch mask, Chen et al. synthesised $\sim 1.5 \mu \mathrm{m}$ long PTFE nanowires on a $50 \mu \mathrm{m}$ thick PTFE film and further utilised it to fabricate a PTFE/Al TENG with an areal power density of $725 \mathrm{mWm}^{-2} \cdot{ }^{11}$ In a sliding mode TENG, the use of ICP RIE to create nanoparticles on the PTFE films was again shown to significantly enhance the power output to nearly $350 \mathrm{~mW} / \mathrm{m}^{2}{ }^{22}$ Although the PTFE thinfilms with relatively high nanostructured surface areas were successfully achieved using the 
ICP-RIE process, the procedure itself is dependent on capital-intensive equipment and processes are complicated and not economically scalable.

In comparison, electrospinning (ES) is an extremely simple and broadly used technique which is capable of producing fibrous nanomaterials with intrinsically high porosity and large surface areas. ${ }^{23}$ Electrospinning relies on the electrostatic force generated between the needle(s) and the collector(s) to produce fine fibres from the charged polymer solution or melt. Since the strong mutual electrical repulsive forces can overcome the weaker forces of surface tension in the charged polymer liquid, the polymer jet can be formed from the charged polymer solution/melt drop, called as Taylor cone, and collected on the collector as fibres with diameters ranging from nanometer $(\mathrm{nm})$ to several micrometres $(\mu \mathrm{m}){ }^{24}$ In previous studies, the technique has already been utilised in the field of TENGs for producing many different polymer membranes with fibrous surface structure, for example, polyvinylidene fluoride (PVDF), ${ }^{17,25}$ Nylon, ${ }^{25}$ ion gel ${ }^{26}$ etc. For these polymer materials, the solvent can be used to dissolve the polymers, so that the corresponding polymer solution can be used for electrospinning process. However, the fabrication of electrospun PTFE nanofibers is much more challenging owing to its high chemical resistance and thermal stability, which means it is nearly impossible to directly obtain/maintain the PTFE solution for electrospinning process. Moreover, since the PTFE does not show any branching behaviour and no chain entanglement, exceptionally high molecular weight $\left(\mathrm{M}_{\mathrm{w}}\right)$ of up to $10,000,000$ $\mathrm{g} / \mathrm{mol}$ is sometimes required to achieve sufficient mechanical strength. ${ }^{27,28}$ However, for such high $\mathrm{M}_{\mathrm{w}}$, the melt viscosity is then too high to process it through melt electrospinning.

To overcome the challenges of using highly toxic solvents and very low solubility of fluorinated polymers, a feasible environment-friendly method for fabricating electrospun fibrous membrane has emerged, called as emulsion electrospinning. ${ }^{29-32}$ The method utilises the mixture of water-based polymer emulsion and water-soluble polymer (carrier) for fabricating composite fibres. After that, a sintering step is performed to process the unspinnable polymer particles into continuous fibres by eliminating the organic carrier. ${ }^{33-35}$ Specifically for electrospinning PTFE, dispersion of PTFE particles in water, stabilised by wetting agents, have been used along with a blend of water-soluble polymer for the preparation of the PTFE fibrous membranes. ${ }^{29,36}$ Reports from Xiong et al. ${ }^{37}$ and Zhou et al., ${ }^{38}$ have utilised polyvinyl alcohol (PVA) as the carrier material to produce PTFE/PVA composite fibres which were further sintered to obtain PTFE nanofibres. Similarly, Feng et al. have utilised polyethene oxide (PEO) as the carrier material to obtain PTFE nanofibres from 
intermediate PTFE/PEO fibres. ${ }^{29}$ It should be mentioned that until now, there have been no reports on the use of electrospun PTFE for applications into TENG devices.

Herein, based on the fabrication of fibrous PTFE porous membranes by electrospinning and polyamide-6 (PA6) membranes by phase inversion process, we report on a cost-effective PTFE based vertical contact-mode TENG with a highly nanostructured rough surface. We further utilise an ion-injection process to introduce a high amount of stable static negative charges on the PTFE surface to significantly enhance the power output. While the average peak-to-peak output voltage (Vp-p) and output current density for the as-sintered ES PTFE/PA6 are $\sim 77 \mathrm{~V}$ and $\sim 1.6 \mathrm{mAm}^{-2}$, corresponding to a charge density of $\sim 13.5 \mu \mathrm{Cm}^{-2}$; after the ion-injection process the corresponding values increase dramatically and stabilise at $\sim 900 \mathrm{~V}, \sim 20 \mathrm{~mA}^{-2}$, and $\sim 149 \mu \mathrm{Cm}^{-2}$ respectively. The electrospun, ion-injected PTFE/PA6 TENG is able to deliver an output power of $\sim 9 \mathrm{Wm}^{-2}$, significantly higher than the values reported using the plasma processed PTFE nanowires-based TENGs. The static negative charges introduced on the surface of PTFE are highly stable in nature as measured using electrostatic force microscopy (EFM) and stability measurements carried out for nearly 30,000 cycles. The work thus introduces a relatively simple, safe and cost-effective route to obtain PTFE nanofiber membranes via emulsion electrospinning. The further ion-injection process enables high surface charge density PTFE based triboelectric nanogenerators for energy harvesting applications and self-powered systems.

\section{Experimental}

\section{Materials}

The aqueous dispersion of PTFE (62 wt\%) was purchased from Dongguan Jin Ming plastic raw material Co. Ltd. (China), with an average PTFE particle size of $80 \mathrm{~nm}$. The values of kinematic viscosity, density and $\mathrm{pH}$ value of the PTFE dispersion (at $25{ }^{\circ} \mathrm{C}$ ) are in the ranges of 6-14 $\mathrm{mm}^{2} \mathrm{~s}^{-1}, 1.48-1.53 \mathrm{gcm}^{-3}$ and 8-10, respectively. Polyethylene oxide (PEO, WAR-205) with a molecular weight $\left(\mathrm{M}_{\mathrm{w}}\right)$ of approximately $600,000 \mathrm{gmol}^{-1}$ was obtained from BDH Chemicals. Polyamide-6 (TECHNYL $1011 \mathrm{R}$ ) was obtained from Rhodia Ltd. The electrospinning solutions were prepared at various different weight ratios of PEO:PTFE with complete details provided in Table 1. 
Table 1. Preparation and composition of electrospinning solutions

\begin{tabular}{llll}
\hline $\begin{array}{l}\text { PEO } \\
(\mathbf{g})\end{array}$ & $\begin{array}{l}\text { Deionized Water } \\
(\mathbf{m l})\end{array}$ & $\begin{array}{l}\mathbf{6 2} \mathbf{w t} \% \mathbf{P T F E} \\
\mathbf{( g )}\end{array}$ & $\begin{array}{l}\text { PEO/PTFE } \\
(\mathbf{w t} / \mathbf{w t})\end{array}$ \\
\hline $\mathbf{0 . 2 9}$ & 4.37 & 9.68 & 0.04 \\
$\mathbf{0 . 4 2}$ & 3.88 & 9.68 & 0.07 \\
$\mathbf{0 . 5 6}$ & 3.74 & 9.677 & 0.09 \\
$\mathbf{0 . 5 5}$ & 2.45 & 7.00 & 0.12 \\
\hline
\end{tabular}

\section{Electrospinning and sintering of PEO-PTFE membranes}

Electrospinning of composite PEO-PTFE membranes was carried out in a home-built electrospinning setup incorporating a high voltage DC supply (Spellman RHR20PN30 Medium Power HV, 0-20 kV), a syringe pump (Aladdin Infusion Pump AL300-220) and a stainless-steel mesh as the collector. All the electrospinning processing runs were performed at an applied voltage of $15 \mathrm{kV}$, collection distance of $\sim 15 \mathrm{~cm}$ and a solution flow rate of 0.5 $\mathrm{mLh}^{-1}$ with a deposition time of $20 \mathrm{~min}$.

The as-obtained electrospun PEO-PTFE membranes were further sintered to obtain pristine PTFE membranes. The sintering process was carried out in a high-temperature tube furnace (ELITE TSH12/100/750-2216) in a flowing nitrogen atmosphere at various temperatures (350, 360 and $370{ }^{\circ} \mathrm{C}$ ) for $15 \mathrm{~min}$ after which the furnace was gradually reduced to the room temperature $\left(\sim 20^{\circ} \mathrm{C}\right)$ in $8 \mathrm{hr}$ in a pure $\mathrm{N}_{2}$ atmosphere. Figure S1 in Supporting Information (SI) is the photos of PTFE membranes before and after sintered at various temperatures.

\section{Fabrication of TENGs}

The physical structure of the contact-mode TENG is based on the conventional design shown in our earlier works. ${ }^{39}$ The PA6 membrane was prepared using a phase inversion process wherein the $20 \mathrm{wt} \%$ dope solution was prepared using formic acid by continuously stirring at approximately $70{ }^{\circ} \mathrm{C}$ for three hours. Further details on the spin-coating process and quenching temperature can be found in our earlier works. ${ }^{39}$ Figure S2 in SI shows the surface and cross-sectional morphology, displaying high porosity of the PA6 membrane used in this work. The obtained PA6 membranes were attached to the acrylic sheet using a conductive adhesive aluminium tape electrode. For the bottom contact layer, the porous electrospun PTFE membranes were again attached using a conductive aluminium tape. Two square 
acrylic sheets of $3 \mathrm{~cm} \times 3 \mathrm{~cm}$ were used as the support substrate, and each of the membranes with an effective area of $2 \mathrm{~cm} \times 2 \mathrm{~cm}$ was fixed at its centre.

\section{Characterisation}

A Hitachi S3400N scanning electron microscope (SEM) was used to observe the surface of PTFE, PA6 membranes and nanofiber morphology. Differential scanning calorimetry (DSC) was used to investigate the crystallinity as well as the presence of PEO in the PTFE membranes using a TA Instruments DSC Q2000. The samples, approximate weight of $2 \mathrm{mg}$, were heated at $10{ }^{\circ} \mathrm{C} \cdot \mathrm{min}^{-1}$ from 0 to $350{ }^{\circ} \mathrm{C}$ under $50 \mathrm{ml} \cdot \mathrm{min}^{-1} \mathrm{~N}_{2}$ flow. The thermogravimetric analysis of the samples was carried out in the range of $25-700{ }^{\circ} \mathrm{C}$ at a ramp rate of $10{ }^{\circ} \mathrm{C} \cdot \mathrm{min}^{-1}$ in a nitrogen atmosphere, using the simultaneous DTA-TGA DT 2960. The FTIR spectra of samples were obtained with a Thermo Scientific IS10 Nicolet at a nominal resolution of $\pm 1 \mathrm{~cm}^{-1}$ for a total of 128 scans and the data obtained was analysed using the vendor-provided software, OMINIC. X-ray photoelectron spectroscopy (XPS) data were obtained on an AXIS Nova Spectrometer (Kratos Analytical Ltd., UK) utilising a monochromatic $\mathrm{Al} K \alpha \mathrm{X}$-ray source (excitation energy of $1486.6 \mathrm{eV}$ ). For binding energy calibration, the signal was calibrated to the F-C-F peak in the high-resolution C1s spectra at $292.8 \mathrm{eV}$ or the F1s signal at $689 \mathrm{eV}$.

The spatial distribution of charges in the PTFE and PA6 membranes was investigated using the electric force microscopy (EFM). EFM experiments were performed at ambient conditions using an MFP3D-Atomic Force Microscope (Asylum Research, Santa Barbara, CA, USA). In the two-step EFM mode, the topographic image was obtained during the main scan in the tapping mode. During interleave (lift) mode, the probe was raised above the sample surface allowing the imaging of relatively weak but long-range electrostatic interactions while minimising the influence of topography. For the EFM imaging, we have used electrically conducting Pt/Ir coated Si probe (AC240 Electrilever, Olympus Valley, PA, USA) with a resonance frequency of $70 \mathrm{kHz}$ and spring constant of $2 \mathrm{~N} / \mathrm{m}$.

The electrical measurements of the assembled TENG devices were carried out using a combination of dynamic fatigue tester system (Popwil Model YPS-1), an oscilloscope (Tektronix MDO3022), and a pico-ammeter (Keysight B2981A). The fatigue test system, interfaced to a personal computer, performs repeated pressing/releasing in the $10-1000 \mathrm{~N}$ load range at a $1-10 \mathrm{~Hz}$ controlled frequency range. 


\section{Results and discussion}

\section{Optimisation of PTFE:PEO ratio}

As discussed earlier, direct electrospinning of PTFE is inherently difficult due to its high chemical inertness and resistance ${ }^{13,14}$ and high melt viscosity which inhibits the use of melt electrospinning technique. Thus, techniques such as co-axial ${ }^{40}$ and emulsion electrospinning ${ }^{13,38}$ have been developed to prepare PTFE nanofiber membranes. Previously, Xiao et al have utilised a blend of polyvinyl alcohol (PVA) with PTFE followed by a sintering process to obtain PTFE nanofibers. However, it was observed that the high percentage of PVA ( $\sim 30 \mathrm{wt} \%$ ) led to poor tensile properties of the ensuing PTFE nanofibers. It is known that to obtain high-quality continuous nanofiber structure from un-spinnable polymers such as PTFE, the concentration ratio of sacrificial carrier to polymer needs to be tuned carefully. At low concentrations of the carrier polymer, no continuous nanofiber structures can be obtained, whereas high concentrations lead to the poor mechanical properties. ${ }^{29}$ Thus, an optimal value of carrier concentration is required where continuity of the fibres is maintained, and yet the carrier polymer can be eliminated during the subsequent sintering process. In our case, to obtain PTFE membranes with a uniform structure, i.e. displaying a good fibrous structure with the PTFE particles embedded uniformly in a PEO matrix, four different weight ratios $(0.04,0.07,0.09$ and 0.12$)$ of PEO/PTFE were tried. The fibrous membranes collected from these four ratios of PEO/PTFE show a significant difference (Figure 1) in fibre morphology. At the lowest weight ratio of 0.04 , no significant fibre structure could be observed, and the deposited film was full of beads ( $2 \pm 1 \mu \mathrm{m}$ diameter sphere) with an only trace amount of fibre connections amongst them as shown in Figure 1a). Such morphology can be attributed to the small quantities of PEO which are unable to bind the PTFE particles together to form a continuous structure.

Based on the previous research, it is known that with the percentage increase in the carrier, the bead-like structures can be reduced or even completely eliminated. ${ }^{29,38}$ Upon increasing the amount of PEO in the mixture to a weight ratio of 0.07 and eventually to 0.09 (Figure 1b\&c), the PTFE/PEO fibres transform from bead-like structure to more fibrous structures, with fibre diameters of $700 \pm 200 \mathrm{~nm}$ and $900 \pm 200 \mathrm{~nm}$ respectively. In fact, the bead-like structures are fully eliminated with the fibrous structure becoming continuous and uniform when the PEO/PTFE ratio is increased to 0.12 (Figure 1d), resulting in an average fibre diameter of $1100 \pm 200 \mathrm{~nm}$. It should be noted that the PEO/PTFE ratio of 0.12 is significantly 
lower than that reported by Xiao et al (0.42, PVA/PTFE) and then makes it easier to remove the carrier polymer to obtain pure PTFE fibres with superior mechanical properties. ${ }^{37}$ However, as compared to the recent report by Feng et al., our optimal ratio is nearly four times higher which could be due to various factors including PEO solution concentration (18 $w t \%), M_{w}$ of PEO, and variation of electrospinning equipment and parameters. ${ }^{29}$

\section{Optimisation of sintering temperature}

The sintering temperature and process are keys to the formation of phase-pure PTFE nanofibers by eliminating the carrier PEO. To assess the sintering temperature range, we first carried out the TGA and DSC analysis of the pristine PEO and as-prepared ES PTFE/PEO samples. For pristine PEO, the TGA thermogram and its differential (DTG) curve revealed that the single mass-loss step occurs in the range of $320-450{ }^{\circ} \mathrm{C}$, with an onset temperature of $\sim 350{ }^{\circ} \mathrm{C}$ and a residual weight of less than $4 \%$ at $600{ }^{\circ} \mathrm{C}$. For the composite ES PTFE/PEO (0.12 ratio) sample, the thermogram shows an expected two-step mass loss, corresponding to the decomposition of PEO and PTFE phases, respectively. While the onset temperature of the PEO phase in the composite film is similar, the peak weight-loss temperature for the PEO component is lower $\left(\sim 385^{\circ} \mathrm{C}\right)$, as compared to the pristine PEO $\left(\sim 405^{\circ} \mathrm{C}\right)$, which can arise

due to the interfacial effects between the PTFE and PEO components. ${ }^{29}$ The second mass loss step occurs in the range of $475-605^{\circ} \mathrm{C}$, corresponding to the decomposition of PTFE, with an onset temperature of $\sim 500{ }^{\circ} \mathrm{C}$.

Now, considering the DSC melting curves for the pristine ES PTFE/PEO sample (Figure 2b), two melting peaks are observed in the range of $40-60{ }^{\circ} \mathrm{C}\left(\mathrm{T}_{\mathrm{M}} \mathrm{PEO}, 38.6{ }^{\circ} \mathrm{C}\right)$ and $310-330{ }^{\circ} \mathrm{C}$ ( $\mathrm{T}_{\mathrm{M}}$ PTFE, $314.6^{\circ} \mathrm{C}$ ), corresponding to the melting of PEO and PTFE phases, respectively. Looking at these results, in a combination of the TGA results, it can be safely assumed that above sintering temperatures of $350{ }^{\circ} \mathrm{C}$, the PEO will start to decompose while the DSC curve indicates that the PTFE will be in molten state $\left(\mathrm{T}_{\mathrm{M}} 314.6^{\circ} \mathrm{C}\right)$. Thus, $350{ }^{\circ} \mathrm{C}$ can be considered as the lower limit of the sintering temperature range. Similarly, the PTFE component of the composite (which is in the molten state above its $\mathrm{T}_{\mathrm{M}}$ ) is stable up to $\sim 450$ ${ }^{\circ} \mathrm{C}$ beyond which its degradation begins and as such represents the higher sintering temperature threshold. Therefore, the sintering temperature range is concurred to be in the range of $350-450{ }^{\circ} \mathrm{C}$, wherein the PEO would have decomposed while the molten PTFE particles could have fused together to form a continuous structure. 
Consequently, the ES PTFE/PEO samples were subjected to a sintering process at a fixed heating rate of $10{ }^{\circ} \mathrm{C} / \mathrm{min}$ from room temperature to the first target temperature of $350{ }^{\circ} \mathrm{C}$. From the SEM images shown in Figure 1d and Figure 3a, it can be observed that the PTFE particles are embedded uniformly in the PEO matrix. Upon heating to $350{ }^{\circ} \mathrm{C}$, a significant difference in the microstructure of the PTFE/PEO fibres could be observed, wherein the PTFE particles seem to have melted and fused together, giving rise to groove like structures across the length and diameter of the fibres (Figure 3b). At a still higher sintering temperature of $370{ }^{\circ} \mathrm{C}$, the transverse groove-like structures could still be observed across the fibres with an apparently smoother surface. Moreover, interconnections between the fibres caused due to the melting and reforming of the structure could also be observed. However, due to fibre shrinkage occurring during the sintering process, a significant difference in the resulting film thickness can be observed. Upon sintering at $350{ }^{\circ} \mathrm{C}$, the thickness of PEO/PTFE composite membranes is dramatically reduced from $\sim 80 \mu \mathrm{m}$ to $\sim 15 \mu \mathrm{m}$ (see the cross-sectional images prior and post-sintering of Figures. 3a\&c). While an increase in the sintering temperature from 350 to $370{ }^{\circ} \mathrm{C}$ does not cause any appreciable change in the overall thickness of the film (see Figures. 3c\&e), however, a variation in the film colour, ranging from bright white for pristine PTFE/PEO to slight grey $\left(350{ }^{\circ} \mathrm{C}\right)$ and dark grey $\left(380{ }^{\circ} \mathrm{C}\right)$ was observed for the sintered samples (see Figure S1, Supporting Information). Previous work by Xiao et al., using a 0.42 PVA/PTFE ratio, reported of cracks, inhomogeneity and discontinuity of sintered PTFE fibres due to the decomposition of a significant amount of PVA. ${ }^{37,41}$ In our case, due to the much lower PEO/PTFE ratio (0.12), PTFE particle wrapped inside the PEO fibres were fully melted together without significantly changing the fibre morphology and is in line with the results reported by Feng et al.. ${ }^{29}$

Also, in our case, the removal of PEO from the composite fibres was confirmed using the DSC wherein no PEO melting peaks were observed for sintered samples (Figure 2b). PTFE is a highly crystalline polymer as evidenced by the relatively high crystallinity value $\left(\Delta \mathrm{X}_{\mathrm{c}}\right)$ of nearly $82.6 \%$ for the as-deposited PTFE/PEO composites. Upon sintering, a reduction in the $\Delta \mathrm{X}_{\mathrm{c}}$ was observed with a value of nearly $62.5 \%$ for the samples sintered at $350{ }^{\circ} \mathrm{C}, 58.0 \%$ for samples sintered at $360{ }^{\circ} \mathrm{C}$ and a value of $59.7 \%$ for samples sintered at $370{ }^{\circ} \mathrm{C}$. This reduction in the crystallinity upon sintering is attributed to the melting and recrystallization of PTFE during the sintering process and has been observed previously by Feng et al. as well. ${ }^{29}$ The sintered PTFE nanofibers also exhibit an extensive crystalline transition at $\sim 20^{\circ} \mathrm{C}$ due to a transformation of the Phase II helix crystal structure (below $20^{\circ} \mathrm{C}$ ) first to the Phase IV 
hexagonal crystal structure (above $20^{\circ} \mathrm{C}$ ) and then to the Phase I hexagonal crystal structure (beyond $25^{\circ} \mathrm{C}$ ). ${ }^{42}$ Interestingly, this phase transition was not observed for the composite films and only appeared for the sintered films, hence indicative of the purity of the PTFE nanofibers upon sintering process.

Further confirmation of the removal of PEO by sintering was corroborated using the FTIR and XPS analysis. In the FTIR spectrum of PTFE (shown in Figure 4a), the most prominent peaks are observed at $\sim 1201$ and $1145 \mathrm{~cm}^{-1}$, originating from the C-F bonds of PTFE. ${ }^{43}$ In contrast, the infrared spectrum of PEO is much more complex with bands at around 1341 and $1467 \mathrm{~cm}^{-1}$, corresponding to the vibrations of the $\mathrm{CH}_{2}$ group, with further strong bands observed at 1093 and $960 \mathrm{~cm}^{-1}$, ascribed to the asymmetric CO stretching vibration. ${ }^{44}$ In the region, around $2880 \mathrm{~cm}^{-1}$, the strong band from PEO is attributed to the symmetric and asymmetric CH stretching. ${ }^{45}$ Now, comparing the pristine ES PTFE/PEO and the samples sintered at various temperatures (350, 360 and $370{ }^{\circ} \mathrm{C}$ ), no bands from PEO (PEO band positions marked as * in Figure $4 \mathrm{a}$ ) could be observed, indicating the removal of PEO from the composite fibres, at least not detectable by FTIR and XPS measurements. The presence of oxygen-related functionalities in the as-deposited ES PTFE/PEO composite fibres was also confirmed through XPS analysis (Figures. 4b-d). As shown in Figure 4b, for the pristine ES PTFE/PEO composite sample, the WESS shows a conspicuous peak of O1s at 530 eV. The surface atomic quantification of the pristine ES PTFE/PEO sample provides a value of 47.6, 44.0 and 8.4 at\% for carbon (C), fluorine (F) and oxygen (O), respectively with an F/C ratio of 0.92. The core level C1s signal for the pristine ES PTFE/PEO sample shows the presence of C-C/C-H (at $284.8 \mathrm{eV}$ ) and C-O (at $286.3 \mathrm{eV}$ ) components present in the PEO. Upon sintering at $370{ }^{\circ} \mathrm{C}$, the surface atomic concentrations change to $34.0,65.9$ and 0.1 at $\%$ for $\mathrm{C}$, $\mathrm{F}$ and $\mathrm{O}$, respectively, with a resulting $\mathrm{F} / \mathrm{C}$ ratio of 1.9 , which is very similar to the expected value for pristine PTFE. ${ }^{27,28,46}$ As compared to the pristine ES PTFE/PEO (full-width at halfmaximum, FWHM 1.3), the line width of the C1s spectra in the sintered sample shows a higher value (2.2) which can be attributed to the formation of possible defects in the structure. However, both the C1s and F1s peaks demonstrated no peak shift, which indicates that the oxygen species being removed during the sintering process do not react with the chemically stable PTFE surface. The results show the optimal sintering temperature to remove PEO and form continuous fibrous structures is $\sim 360{ }^{\circ} \mathrm{C}$. Such processed PTFE nanofibers were used to fabricate the TENG devices as shown in the following section.

\section{Electrical characterisation of TENGs}


Considering the triboelectric series, PTFE is one of the strongest tribo-negative materials due to the presence of significant quantities of electronegative fluorine in the structure, whereas the polyamide can readily provide the charge, which is then accepted by the PTFE and as such the system can provide a significantly high power output. ${ }^{10}$ As both the voltage and current outputs of the TENG are proportional to the surface triboelectric charge density, the resulting power output has a quadratic dependence on the surface charge density. As shown in Figures. 5a\&b, the TENG assembled using the PA6 and sintered ES PTFE, under an external force of $50 \mathrm{~N}$ and contact frequency of $5 \mathrm{~Hz}$, produces an open-circuit voltage $\left(\mathrm{V}_{\text {oc }}\right)$ of $\sim 40 \mathrm{~V}$, with a short-circuit peak current density $\left(\mathrm{J}_{\mathrm{sc}}\right)$ of $\sim 1.6 \mathrm{mAm}^{-2}$ (corresponding to a charge density of $13.7 \mu \mathrm{Cm}^{-2}$ ). The values obtained here are very similar to those reported by Yang et al., in their work on triboelectric nanogenerators prepared using thin films of PTFE and PA. ${ }^{47}$ Through the judicious use of materials, surface nanostructuring and further introduction of static surface charges, the power output of the TENGs can be further improved significantly. However, it is observed that, for PTFE based TENGs, the negative charges were required to be introduced to the surface of the PTFE films in advance of the TENG fabrication processes to improve the electrical outputs. The negative charge introduction processes were realised by either a direct high-voltage charge injection ${ }^{5}$ or are the side effect of the RIE process ${ }^{11,22,47,48}$ with relatively high equipment requirement. Herein, we have utilised a commercial anti-static gun (Zerostat 3, Milty) to achieve the same effect through the direct deposition of the negative ions directly onto the ES PTFE surfaces. ${ }^{49}$

The working principle of the anti-static gun is based on the piezoelectric effect which consists of 'two-cycle' operation. A compression trigger and two piezoelectric crystals are embedded inside the gun which generates positive ion flow when the trigger is squeezed and negative ion flow when the trigger is released. ${ }^{50-52}$ By repeating these squeezing-releasing operations, the positive and negative charges can be induced on the target surface. The anti-static gun is usually used for deionization process or preventing electrostatic charge attracting dust onto critical samples/surfaces in the laboratories. ${ }^{51}$ In Bharat's research, the anti-static gun was utilised to deposit the negative charge on the surface of hydrophobic polystyrene to form a negatively charged surface. ${ }^{52}$ In our work, we have deposited negative ions on the assembled ES PTFE/Al layered structure at a vertical distance of $\sim 2 \mathrm{~cm}$ (shown in the inset of Figure $6 a)$. It has been shown previously that during the release cycle, various ions including $\mathrm{CO}_{3}^{-}$, $\mathrm{NO}_{3}^{-}, \mathrm{NO}_{2}^{-}, \mathrm{O}_{3}^{-}$and $\mathrm{O}_{2}^{-}$are injected, making the surface negatively charged. In order to calculate the amount of negative charge injected onto the surface of PTFE surface, the ES 
PTFE layer of the TENG was connected with a coulomb meter (PH0913-N8) through the back $\mathrm{Al}$ electrode. From the measurements, it was observed that an overall charge density of $\sim 150 \mu \mathrm{Cm}^{-2}$ flowed from the ground to the back electrode of membrane for each ion-injection cycle and is indicative of the amount of charge deposited on the PTFE surface. This enhancement in the surface charge density was further confirmed using EFM analysis and is discussed later.

The electrical output measurements of the TENGs, which were carried out at the condition of $50 \mathrm{~N}$ impact force, $5 \mathrm{~Hz}$ contact frequency and $5 \mathrm{~mm}$ spacer distance; before and after the ion-injection process are shown in Figures. 5a\&b. Prior to the ion-injection process, the maximum peak-to-peak voltage (Vp-p), current density and charge density were measured to be $\sim 77 \mathrm{~V}, \sim 1.6 \mathrm{mAm}^{-2}$ and $\sim 13.5 \mu \mathrm{Cm}^{-2}$, respectively. After the ion injection process, corresponding values are dramatically increased to $\sim 960 \mathrm{~V}, \sim 25 \mathrm{mAm}^{-2}$ and $\sim 160 \mu \mathrm{Cm}^{-2}$ (see insets Figure 5a-ii, Figure 5b-ii and Figure S3, Supporting Information) which eventually stabilise to $\mathrm{V}_{\mathrm{p} \text {-p }}$ value of $900 \mathrm{~V}$, current density of $\sim 22 \mathrm{mAm}^{-2}$ and corresponding charge density of $\sim 149 \mu \mathrm{Cm}^{-2}$, nearly 12, 14 and 11 times higher than those of the pristine ES PTFE sample. However, with further charge-injection steps, there is no further electrical output enhancement being observed. Thus, it is reasonable to assume that, after the first-time ioninjection process, the charge on the surface of ES PTFE had already reached its maximum surface density. This behaviour is significantly different from the one observed by Wang et al. in their work on charge injection in fluorinated ethylene propylene (FEP). ${ }^{49}$ In the case of the electret FEP, the presence of voids within the FEP structure allows for a significantly higher density of charges to be deposited onto the surface, unlike the electrospun PTFE membranes which do not have similar porosity.

The working mechanism of the dielectric-to-dielectric contact TENG is based on the triboelectric effect induced electrostatic charges on the PTFE and PA6 films. ${ }^{19}$ When two different tribo-materials are brought into contact, a contact-charge exchange will occur to maintain the electrostatic equilibrium state on the interface. When a material (donor) contacts with the negatively charged surface, to achieve the electrostatic equilibrium state on the contact interface, it will tend to lose more electrons, which will lead a higher density of positive charge on the surface after separating. According to Niu. et al, the transferred charge of the TENGs are directly proportional to the surface charge density of the contact materials. ${ }^{19}$ Thus, the deposition of the negative charge on the surface of the material will dramatically increase the transferred surface charge density on both sides of the contact 
surfaces. As the value of transferred charge density is enhanced via ion-injection, it causes the induced electric potential established between the two electrodes on the back of the triboelectric materials to increase to a higher-level due to electrostatic induction, which leads to higher output performance.

Figure 5c\&d show the output voltage and current measurements for the ion-injected ES PTFE/PA6 TENG operated at various external forces from 20-80 $\mathrm{N}$ at a fixed driving frequency $(5 \mathrm{~Hz})$ and spacer distance $(4 \mathrm{~mm})$. It can be clearly observed that even at relatively low force $(20 \mathrm{~N})$ conditions, the ES PTFE/PA6 TENG provided an open circuit voltage and current density of $\sim 400 \mathrm{~V}$ and $15 \mathrm{~mA} / \mathrm{m}^{2}$. With an increase in the impact force, both the electrical output parameters increase almost linearly with the applied impact force.

The output powers of the ion-injected ES PTFE/PA6 TENG at working condition of $50 \mathrm{~N}, 5$ $\mathrm{Hz}$ impact frequency were measured by connecting the TENG to different load resistors (Figure 6a). Displaying the classical behaviour for maximum transfer power, the current amplitude drops with the increase of load resistance owing to the Ohmic losses. The instantaneous peak power $\left(\mathrm{P}=\mathrm{I}^{2} \mathrm{R}\right)$ is maximised at a load resistance of $200 \mathrm{M} \Omega$, corresponding to a peak power density of $\sim 9 \mathrm{Wm}^{-2}$.

To further study the stability of such ion-injected TENGs, we operated the ES PTFE/PA6 TENG for over 30,000 energy generation cycles at a working frequency of $5 \mathrm{~Hz}$. Within the 100-minute operation time, no attenuation of the $V_{\text {oc }}$ values could be observed, thus indicating that the ion-injection process is an effective practical method for fabricating highpower output TENGs. In addition, the sintering process not only helps to eliminate the carrier but also lead to much higher mechanical properties which can help sintered membranes based TENGs to maintain the surface structure under high-pressure compressions and provide highly stable electrical outputs during the long-term resistance test. ${ }^{29}$ It is necessary to mention that, in order to initialise the dynamic fatigue tester system, the ion-injected PTFE membrane layer needs to be kept in contact with the PA6 membrane for several minutes for calibrating the impact position before the TENG electrical output test. Thus, the negative charges can stay stable on the surface of dielectric materials even after keep contacting with other dielectric materials.

Apart from the deposition of negative-ion on the electrospun PTFE membrane surface, an additional positive ion-injection operation was applied to the positive tribo-material surface (PA6 membrane) to further enhance the TENG output. However, compared to the negative 
ion-injection process on the PTFE surface, the effect of the positive ion-injection to PA6 surface on the output performance of the TENG is negligible. It can be observed from Figure S6 in SI, that the positive injection merely provided a negligible instant voltage output improvement ( 10 V). Furthermore, the stability of injected charge was quite poor wherein the injected charge was eliminated within the 200 -second continuous test.

To investigate the stored charge on the PTFE and PA6 membranes, EFM experiments were conducted. EFM operates in a typical "lift-mode" and is based on the fact that an oscillating cantilever is sensitive to long-range electrostatic force gradients. Variation in probe-sample capacitance (C) created by stored charges (q) or surface charges in the sample is detected by a dual-pass measurement approach. During the first pass scan, the conductive probe in standard tapping mode measures topography. In a second "nap” pass, the topography data allows the probe to retrace the scan line at a constant height or a fixed height (lift height $z$ ) above the surface. Increasing the lift scan height or separating the probe and sample enhances sensitivity to long-range electrostatic forces, while maintaining constant height minimizes effects from other interactions such as van der Waals forces. During the nap pass, the cantilever is excited to its resonance frequency, and a DC voltage, $\mathrm{V}_{\mathrm{EFM}}$, is applied between the probe and the sample. If the surface of the sample is homogeneous and no electric charges trapped, the EFM signal is uniform and constant all over the surface. However, the presence of trapped or stored electric charges would shift the resonance frequency of the cantilever. The resulting changes in the amplitude and phase of the cantilever at the oscillation frequency are recorded in the EFM amplitude and phase channels. The use of phase measurement in EFM is a direct and fast way to detect qualitatively electrostatic force gradients. This change in phase or phase shift of the cantilever is given as: ${ }^{39}$

$$
\Delta \varphi=-\frac{\mathrm{Q}}{\mathrm{k}} \frac{\partial \mathrm{F}}{\partial \mathrm{z}}\left(\mathrm{z}_{0}\right)=-\frac{\mathrm{Q}}{2 \mathrm{k}} \frac{\mathrm{d}^{2} \mathrm{C}}{\mathrm{dz}^{2}}\left(\mathrm{~V}_{\mathrm{EFM}}-\mathrm{V}_{\mathrm{q}}\right)^{2}
$$

Where $\mathrm{Q}$ is the quality factor, $\mathrm{k}$ is the spring constant of EFM cantilever, and $\mathrm{z}_{0}$ is the cantilever equilibrium position, $\mathrm{F}$ is the electrostatic force experienced by the probe and $\mathrm{V}_{\mathrm{q}}$ is the surface potential due to q charges.

Figure 7a-c shows the topography, EFM amplitude and EFM phase signal of the uncharged sintered PTFE, respectively, while Figure 7d-f shows the topography, EFM amplitude and EFM phase signal of the charged PTFE. The amplitude signal is significantly enhanced in the charged PTFE sample (Figure 7e) as compared to uncharged PTFE sample (Figure 7b). The 
contrast phase and amplitude images demonstrate the heterogeneity of the charge distribution in the samples. These images allow the identification of specific areas characterised for the absence or presence of charge. However, EFM phase measurements are more sensitive to the distribution of charge in the sample than amplitude measurements.

The phase measurement contains information about the direction of the electrostatic force (i.e. polarity of charges), On the other hand, the amplitude difference is only an indication of the presence of charge, regardless of its polarity. The EFM image acquired with $\mathrm{V}_{\mathrm{EFM}}=3 \mathrm{~V}$ before charge injection is shown in Figure 7c, revealing the presence of both positive and negative charges for the uncharged ES PTFE sample. If $\mathrm{V}_{\mathrm{EFM}}$ has the opposite sign of $\mathrm{V}_{\mathrm{q}}$, the force gradient decreases and dark features appear in the EFM phase image. The EFM signal here is the cantilever phase shift $\Delta \varphi$ required to maintain a constant cantilever phase in the EFM pass. In the absence of trapped or stored charge, the phase shift is due to the variation of the probe-sample capacitance during the linear pass of the probe over the sample. For fluorinated polymers (such as PTFE), it is expected that the samples would show a highly negative surface owing to the electronegative fluorine in the structure. Comparatively, the polymers containing oxygen groups (such as PEO) would show positively charged and positive surface potential. ${ }^{53}$ Previous studies by Burgo et al. have shown that the PTFE surface consists of macroscopically segregated positive and negative charge domains, rather than exclusive negative charges. ${ }^{54}$ In their report, it has also been argued that the positive macro-domains on PTFE are formed by species derived from contact with polyethylene. This effect can be further demonstrated by observing the EFM scanning result shown in Figure 7i. Without ion-injection, both positive charge domain and negative domains can be detected from the surface of a commercial PTFE film (FLOFILM ${ }^{\mathrm{TM}} 10012.7 \mu \mathrm{m}$ thickness) with a relatively flat surface. By combining the commercial PTFE film with a PA6 membrane as a TENG device, the respective outputs (shown in Figure S4) were observed to be lower than the ES PTFE based TENG without ion-injection process. After being treated with the similar ion-injection process, the output performance of the commercial flat PTFE film based TENG is significantly lower than the electrospun PTFE based one at the same working conditions (shown in Figure S5 in SI). In particular, the charge density of the electrospun PTFE TENG $\left(\sim 149 \mu \mathrm{Cm}^{-2}\right)$ is almost twice that of the flat PTFE film one $\left(\sim 85 \mu \mathrm{Cm}^{-2}\right)$, which is attributed to the much higher effective surface area of the contact interface, even though the surface patterns of the PA6 surface and the electrospun PTFE surface are not fully geometrically matched yet. 
In our case, before the sintering process, the existence of both positive and negative domains on ES PTFE-PEO composite fibre surface can be confirmed by observing Figure S7c. Although crystalline phase PEO segments have been decomposed and removed through the sintering process, isolated residual segments may still exist on the PTFE nanofibers. These residual PEO segments can induce a net positive charge on the surface of the sintered PTFE as visible in Figure 7c, which may contribute to residual positive charge on the sintered PTFE surface alongside the negative charge. This inference is supported by the enhancement of the $\mathrm{C}-\mathrm{H}$ stretching band in the $2800-3000 \mathrm{~cm}^{-1}$ region in the FTIR spectrum, which can only come from the species derived from PEO as the PTFE with its $\mathrm{CF}_{2}-\mathrm{CF}_{2}$ structure should not show any C-H stretching (Figure 7h). Similar results have also been shown by Burgo et al. in their work on the identification of charged species in PTFE/PE system. ${ }^{54}$ The presence of positive charges on the sintered PTFE sample and commercial PTFE sample can also potentially explain the poor electrical response from the fabricated TENGs prior to the charge-injection process. When the sample is charged by the ion-injection process, the electrospun fibres in the sample show higher contrast or larger phase shift (Figure 7f), due to higher force gradient, which physically corresponds to the attractive interaction between the stored charge (i.e. negative charges) and capacitive charges (positive charges) at the EFM tip. Figure $7 \mathrm{~g}$ shows the bias dependent (with attractive and repulsive tip potentials) EFM results on the charged ES PTFE-PA6 sample. The parabola fitting curve (black dash lines) matches well with the experimental data points and confirms that the choice of $+4 \mathrm{~V}$ and $-4 \mathrm{~V}$ as the maxima of the sweeping voltage keeps the data points within a parabolic regime. Thus, the EFM measurements confirm the neutralised positive domain and enhanced negative charge density on the ES PTFE surfaces upon ion-injection treatment.

\section{Conclusion}

High purity PTFE fibrous membranes with the large surface area were successfully fabricated by emulsion electrospinning technique followed by a high-temperature sintering process. Combining with the phase inversion PA6 membranes, the fabricated PTFE fibrous membranes were further applied for producing TENG devices. Subsequently, a simple negative ion-injection process was utilised not only to neutralise the positive charge introduced by the PEO with oxygen groups, but also dramatically enhance the surface negative charge density of the PTFE membranes. The electrospun PTFE fibrous membranes based TENG exhibits a remarkably high output performance when contacted at a $5 \mathrm{~Hz}$ 
working frequency, the device gave a stable peak-to-peak open-circuit voltage of $900 \mathrm{~V}$ and short-circuit current density of $20 \mathrm{mAm}^{-2}$, corresponding to a current density of $\sim 149 \mu \mathrm{Cm}^{-2}$. In addition, a maximum power density of $\sim 9 \mathrm{~W} / \mathrm{m}^{2}$ was obtained at an impedance matching condition with high stability and reliability. The work unambiguously shows a simple, costeffective, and environmentally-friendly technique for fabricating PTFE fibrous membranes and fully demonstrates their great application potential in the field of energy harvesting.

\section{Acknowledgements}

Materials support from Polyflon Technology Ltd. (UK) and Zeus Industrial Products is greatly acknowledged. We would also like to thank the National EPSRC XPS User's Service (NEXUS) at Newcastle University, an EPSRC Mid-Range Facility, for the carrying out X-ray photoelectron measurements. This work was partially supported by the National Natural Science Foundation of China (61471233).

\section{Supporting Information}

The supporting information is available free of charge on the ACS publications website.

Digital photographs of unsintered, sintered electrospun PTFE films; SEM images of PA6 membranes; electrical output of pristine electrospun TENG before and after ion-injection; electrical output of commercial PTFE thin films before and after ion-injection; effect of positive ion-injection on PA6 membrane; EFM analysis of electrospun PTFE/PEO composite membranes prior to sintering. 


\section{References}

(1) Fan, F. R.; Tian, Z. Q.; Lin Wang, Z. Flexible Triboelectric Generator. Nano Energy 2012, 1 (2), 328-334.

(2) Wang, Z. L. Triboelectric Nanogenerators as New Energy Technology for SelfPowered Systems and as Active Mechanical and Chemical Sensors. ACS Nano 2013, 7 (11), 9533-9557.

(3) Yang, W.; Chen, J.; Zhu, G.; Yang, J.; Bai, P.; Su, Y.; Jing, Q. Harvesting Energy from the Natural Vibration of Human Walking. 2013, No. 12, 11317-11324.

(4) Zhong, J.; Zhong, Q.; Fan, F.; Zhang, Y.; Wang, S.; Hu, B.; Wang, Z. L.; Zhou, J. Finger Typing Driven Triboelectric Nanogenerator and Its Use for Instantaneously Lighting up LEDs. Nano Energy 2013, 2 (4), 491-497.

(5) Jiang, T.; Yao, Y.; Xu, L.; Zhang, L.; Xiao, T.; Wang, Z. L. Spring-Assisted Triboelectric Nanogenerator for Efficiently Harvesting Water Wave Energy. Nano Energy 2017, 31, 560-567.

(6) Wu, C.; Liu, R.; Wang, J.; Zi, Y.; Lin, L.; Wang, Z. L. A Spring-Based Resonance Coupling for Hugely Enhancing the Performance of Triboelectric Nanogenerators for Harvesting Low-Frequency Vibration Energy. Nano Energy 2017, 32, 287-293.

(7) Zi, Y.; Lin, L.; Wang, J.; Wang, S.; Chen, J.; Fan, X.; Yang, P. K.; Yi, F.; Wang, Z. L. Triboelectric-Pyroelectric-Piezoelectric Hybrid Cell for High-Efficiency EnergyHarvesting and Self-Powered Sensing. Adv. Mater. 2015, 27 (14), 2340-2347.

(8) Bae, J.; Lee, J.; Kim, S.; Ha, J.; Lee, B. S.; Park, Y.; Choong, C.; Kim, J. B.; Wang, Z. L.; Kim, H. Y.; Park, J. J.; Chung, U. I. Flutter-Driven Triboelectrification for Harvesting Wind Energy. Nat Commun 2014, 5, 4929.

(9) Seol, M. L.; Woo, J. H.; Jeon, S. B.; Kim, D.; Park, S. J.; Hur, J.; Choi, Y. K. Vertically Stacked Thin Triboelectric Nanogenerator for Wind Energy Harvesting. Nano Energy 2015, 14, 201-208.

(10) Wang, Z. L. Triboelectric Nanogenerators as New Energy Technology and SelfPowered Sensors - Principles, Problems and Perspectives. R. Soc. Chem. 2014, 0 (11), $1-12$.

(11) Chen, J.; Zhu, G.; Yang, W.; Jing, Q.; Bai, P.; Yang, Y.; Hou, T. C.; Wang, Z. L. Harmonic-Resonator-Based Triboelectric Nanogenerator as a Sustainable Power Source and a Self-Powered Active Vibration Sensor. Adv. Mater. 2013, 25 (42), 60946099.

(12) Chen, J.; Yang, J.; Li, Z.; Fan, X.; Zi, Y.; Jing, Q.; Guo, H.; Wen, Z.; Pradel, K. C.; Niu, S.; Wang, Z. L. Networks of Triboelectric Nanogenerators for Harvesting Water Wave Energy: A Potential Approach toward Blue Energy. ACS Nano 2015, 9 (3), 3324-3331.

(13) Huang, Q. lin; Xiao, C. fa; Hu, X. yu; Li, X. feng. Study on the Effects and Properties of Hydrophobic Poly(tetrafluoroethylene) Membrane. Desalination 2011, 277 (1-3), 187-192.

(14) Wang, X. Theoretical and Experimental Studies on Acetylene Absorption in a Polytetrafluoroethylene Hollow-Fiber Membrane Contactor. 2015, No. 0, 1-9. 
(15) Wang, S.; Xie, Y.; Niu, S.; Lin, L.; Wang, Z. L. Freestanding Triboelectric-LayerBased Nanogenerators for Harvesting Energy from a Moving Object or Human Motion in Contact and Non-Contact Modes. Adv. Mater. 2014, 26 (18), 2818-2824.

(16) Zhang, H.; Yang, Y.; Zhong, X.; Su, Y.; Zhou, Y.; Hu, C.; Wang, Z. L. SingleElectrode-Based Rotating Triboelectric Nanogenerator for Harvesting Energy from Tires. ACS Nano 2014, 8 (1), 680-689.

(17) Cui, S.; Zheng, Y.; Liang, J.; Wang, D. Conducting Polymer PPy Nanowire-Based Triboelectric Nanogenerator and Its Application for Self-Powered Electrochemical Cathodic Protection. Chem. Sci. 2016, 7 (10), 6477-6483.

(18) Niu, S.; Wang, Z. L. Theoretical Systems of Triboelectric Nanogenerators. Nano Energy 2014, 14, 161-192.

(19) Niu, S.; Wang, S.; Lin, L.; Liu, Y.; Zhou, Y. S.; Hu, Y.; Wang, Z. L. Theoretical Study of Contact-Mode Triboelectric Nanogenerators as an Effective Power Source. Energy Environ. Sci. 2013, 6 (12), 3576.

(20) Md Jani, A. M.; Losic, D.; Voelcker, N. H. Nanoporous Anodic Aluminium Oxide: Advances in Surface Engineering and Emerging Applications. Prog. Mater. Sci. 2013, 58 (5), 636-704.

(21) Zhang, B.; Chen, J.; Jin, L.; Deng, W.; Zhang, L.; Zhang, H.; Zhu, M.; Yang, W.; Wang, Z. L. Rotating-Disk-Based Hybridized Electromagnetic- Triboelectric Nanogenerator for Sustainably Powering Wireless Traffic Volume Sensors. ACS Nano, 2016, 10 (6), 6241-6247.

(22) Yang, Y.; Zhang, H.; Chen, J.; Jing, Q.; Zhou, Y. S.; Wen, X.; Wang, Z. L. SingleElectrode-Based Sliding Triboelectric Nanogenerator for Self-Powered Displacement Vector Sensor System. ACS Nano 2013, 7 (8), 7342-7351.

(23) Xie, J.; Xia, Y. Electrospinning: An Enabling Technique for Nanostructured Materials. Mater. Matters 2008, 3, 19-23.

(24) Bhardwaj, N.; Kundu, S. C. Electrospinning: A Fascinating Fiber Fabrication Technique. Biotechnol. Adv. 2010, 28 (3), 325-347.

(25) Zheng, Y.; Cheng, L.; Yuan, M.; Wang, Z.; Zhang, L.; Qin, Y.; Jing, T. An Electrospun Nanowire-Based Triboelectric Nanogenerator and Its Application in a Fully Self-Powered UV Detector. Nanoscale 2014, 6 (14), 7842-7846.

(26) Ye BU, Kim BJ, Ryu J, Lee JY, Baik JM, H. K. Electrospun Ion Gel Nanofibers for Flexible Triboelectric Nanogenerator Electrochemical Effect on Output Power. Nanoscale 2015, 7, 16189-16194.

(27) Hunke, H.; Soin, N.; Shah, T.; Kramer, E.; Pascual, A.; Karuna, M.; Siores, E. LowPressure H2, NH3 Microwave Plasma Treatment of Polytetrafluoroethylene (PTFE) Powders: Chemical, Thermal and Wettability Analysis. Materials (Basel). 2015, 8 (5), 2258-2275.

(28) Hunke, H.; Soin, N.; Shah, T.; Kramer, E.; Witan, K.; Siores, E. Influence of Plasma Pre-Treatment of Polytetrafluoroethylene (PTFE) Micropowders on the Mechanical and Tribological Performance of Polyethersulfone (PESU)-PTFE Composites. Wear 2015, 328-329, 480-487. 
(29) Feng, Y.; Xiong, T.; Jiang, S.; Liu, S.; Hou, H. Mechanical Properties and Chemical Resistance of Electrospun Polyterafluoroethylene Fibres. RSC Adv. 2016, 6 (29), 24250-24256.

(30) Hu, J.; Prabhakaran, M. P.; Tian, L.; Ding, X.; Ramakrishna, S. Drug-Loaded Emulsion Electrospun Nanofibers: Characterization, Drug Release and in Vitro Biocompatibility. Rsc Adv. 2015, 5 (121), 100256-100267.

(31) Yazgan, G.; Popa, A. M.; Rossi, R. M.; Maniura-Weber, K.; Puigmartí-Luis, J.; Crespy, D.; Fortunato, G. Tunable Release of Hydrophilic Compounds from Hydrophobic Nanostructured Fibers Prepared by Emulsion Electrospinning. Polym. (United Kingdom) 2015, 66, 268-276.

(32) Agarwal, S.; Greiner, A. On the Way to Clean and Safe Electrospinning-Green Electrospinning: Emulsion and Suspension Electrospinning. Polym. Adv. Technol. 2011, 22 (3), 372-378.

(33) Puppi, D.; Piras, a. M.; Detta, N.; Ylikauppila, H.; Nikkola, L.; Ashammakhi, N.; Chiellini, F.; Chiellini, E. Poly(vinyl Alcohol)-Based Electrospun Meshes as Potential Candidate Scaffolds in Regenerative Medicine. J. Bioact. Compat. Polym. 2011, 26 (1), 20-34.

(34) Bansal, P.; Bubel, K.; Agarwal, S.; Greiner, A. Water-Stable All-Biodegradable Microparticles in Nanofibers by Electrospinning of Aqueous Dispersions for Biotechnical Plant Protection. Biomacromolecules 2012, 13 (2), 439-444.

(35) Giebel, E.; Mattheis, C.; Agarwal, S.; Greiner, A. Chameleon Nonwovens by Green Electrospinning. Adv. Funct. Mater. 2013, 23 (25), 3156-3163.

(36) Huang, Y.; Huang, Q. L.; Liu, H.; Zhang, C. X.; You, Y. W.; Li, N. N.; Xiao, C. F. Preparation, Characterization, and Applications of Electrospun Ultrafine Fibrous PTFE Porous Membranes. J. Memb. Sci. 2017, 523 (399), 317-326.

(37) Xiong, J.; Huo, P. F.; Ko, F. K. Fabrication of Ultrafine Fibrous Polytetrafluoroethylene Porous Membranes by Electrospinning. J. Mater. Res. 2009, 24 (9), 2755-2761.

(38) Zhou, T.; Yao, Y.; Xiang, R.; Wu, Y. Formation and Characterization of Polytetrafluoroethylene Nanofiber Membranes for Vacuum Membrane Distillation. $J$. Memb. Sci. 2014, 453, 402-408.

(39) Soin, N.; Zhao, P.; Prashanthi, K.; Chen, J.; Ding, P.; Zhou, E.; Shah, T.; Ray, S. C.; Tsonos, C.; Thundat, T.; Siores, E.; Luo, J. High Performance Triboelectric Nanogenerators Based on Phase-Inversion Piezoelectric Membranes of Poly(vinylidene Fluoride)-Zinc Stannate (PVDF-ZnSnO3) and Polyamide-6 (PA6). Nano Energy 2016, 30, 470.

(40) Muthiah, P.; Hsu, S.; Sigmund, W. Coaxially Electrospun PVDF - Teflon AF and Teflon AF - PVDF Core - Sheath Nanofiber Mats with Superhydrophobic Properties. 2010, 26 (11), 12483-12487.

(41) Ahmed, F. E.; Lalia, B. S.; Hashaikeh, R. A Review on Electrospinning for Membrane Fabrication: Challenges and Applications. Desalination 2015, 356, 15-30. 
(42) Breiby, D. W.; Sølling, T. I.; Bunk, O.; Nyberg, B.; Norrman, K.; Nielsen, M. M. Structural Surprises in Friction-Deposited Films of Poly ( Tetrafluoroethylene ). 2005, 2383-2390.

(43) Li, L.; Zi, F. T.; Zheng, Y. F. Applied Surface Science The Characterization of Fluorocarbon Films on NiTi Alloy by Magnetron Sputtering. 2008, 255, 432-434.

(44) Sui, X.; Shao, C.; Liu, Y. Photoluminescence of Polyethylene Oxide-ZnO Composite Electrospun Fibers. Polymer (Guildf). 2007, 48 (6), 1459-1463.

(45) Tang, S.; Shao, C.; Liu, Y.; Li, S.; Mu, R. Electrospun Nanofibers of Poly(ethylene Oxide)/teraamino-Phthalocyanine copper(II) Hybrids and Its Photoluminescence Properties. J. Phys. Chem. Solids 2007, 68 (12), 2337-2340.

(46) Vesel, A.; Mozetic, M.; Zalar, A. XPS Characterization of PTFE after Treatment with RF Oxygen and Nitrogen Plasma. Surf. Interface Anal. 2008, 40 (3-4), 661-663.

(47) Zhang, H.; Yang, Y.; Su, Y.; Chen, J.; Hu, C.; Wu, Z.; Liu, Y.; Ping Wong, C.; Bando, Y.; Wang, Z. L. Triboelectric Nanogenerator as Self-Powered Active Sensors for Detecting Liquid/gaseous Water/ethanol. Nano Energy 2013, 2 (5), 693-701.

(48) Yang, P.; Lin, Z.; Wang, Z. L.; Pradel, K. C.; Lin, L.; Li, X.; Wen, X.; He, J.; Science, M.; States, U.; Engineering, E.; Sciences, M.; Arabia, S.; Engineering, B.; Academy, C. Paper-Based Origami Triboelectric. ACS Nano 2015, 9 (1), 901-907.

(49) Wang, S.; Xie, Y.; Niu, S.; Lin, L.; Liu, C.; Zhou, Y. S.; Wang, Z. L. Maximum Surface Charge Density for Triboelectric Nanogenerators Achieved by Ionized-Air Injection: Methodology and Theoretical Understanding. Adv. Mater. 2014, 26 (39), $6720-6728$.

(50) Gates, M. W.; Buffington, M. L. Description of Two Techniques to Increase Efficiency in Processing and Curating Minute Arthropods, with Special Reference to Parasitic Hymenoptera. 2011, 140, 133-140.

(51) Hoe, S.; Traini, D.; Chan, H. K.; Young, P. M. The Contribution of Different Formulation Components on the Aerosol Charge in Carrier-Based Dry Powder Inhaler Systems. Pharm. Res. 2010, 27 (7), 1325-1336.

(52) Bhushan, B.; Pan, Y.; Daniels, S. AFM Characterization of Nanobubble Formation and Slip Condition in Oxygenated and Electrokinetically Altered Fluids. J. Colloid Interface Sci. 2013, 392 (1), 105-116.

(53) Diaz, A. F.; Felix-Navarro, R. M. A Semi-Quantitative Tribo-Electric Series for Polymeric Materials: The Influence of Chemical Structure and Properties. J. Electrostat. 2004, 62 (4), 277-290.

(54) Burgo, T. A. L.; Ducati, T. R. D.; Francisco, K. R.; Clinckspoor, K. J.; Galembeck, F.; Galembeck, S. E. Triboelectricity: Macroscopic Charge Patterns Formed by SelfArraying Ions on Polymer Surfaces. Langmuir 2012, 28 (19), 7407-7416. 

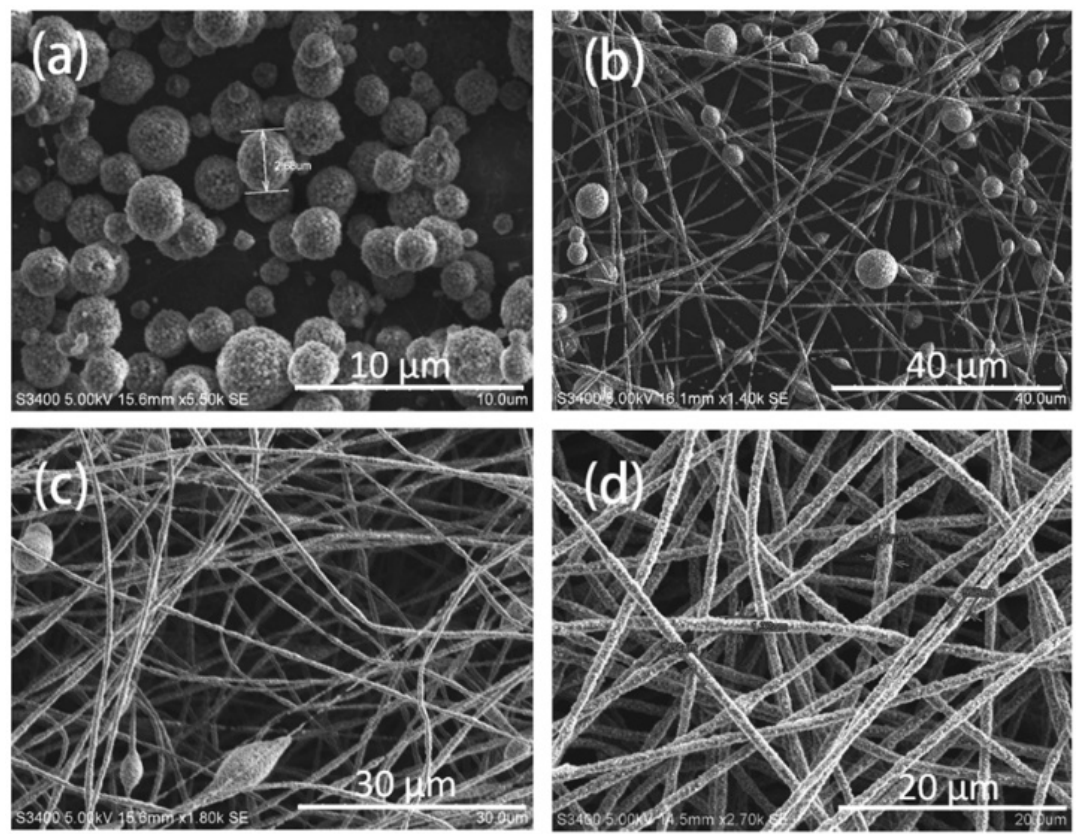

Figure 1. SEM images of the electrospun PEO/PTFE composite fibres at different PEO:PTFE weight ratios of (a) 0.04 , (b) 0.07 , (c) 0.09 and (d) 0.12 .
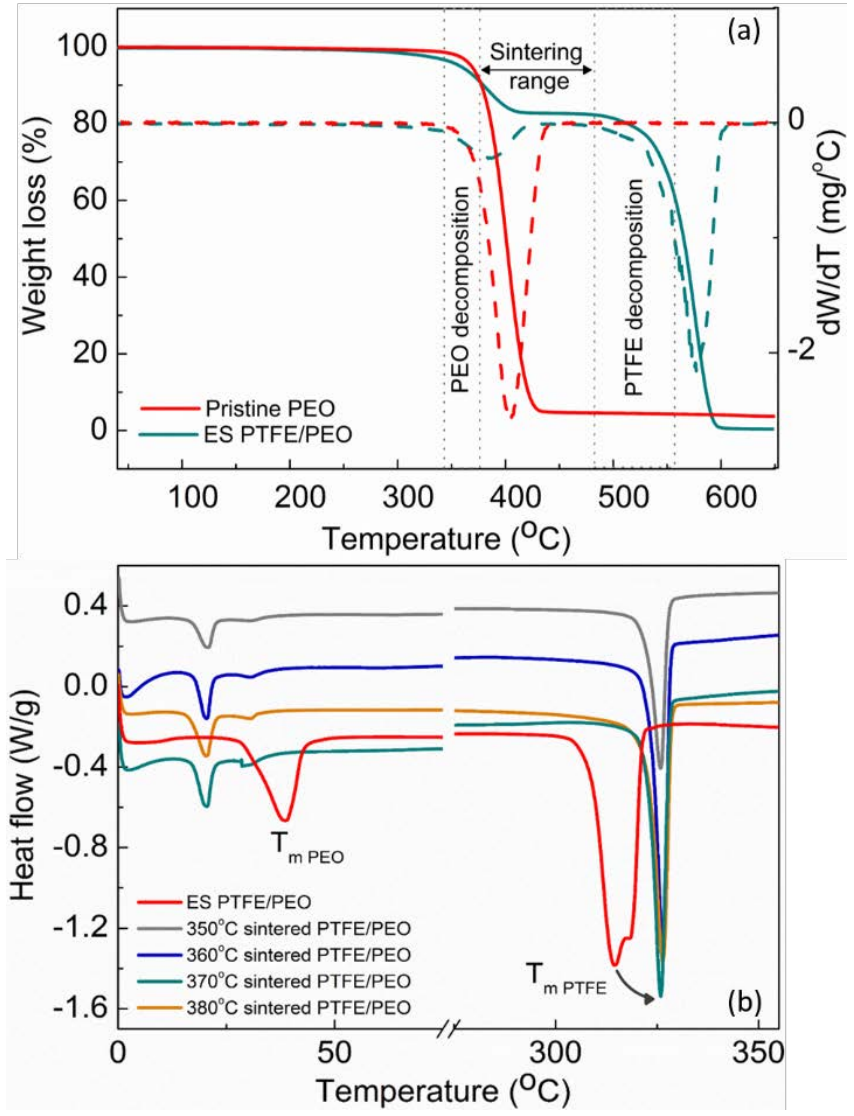

Figure 2. (a) TGA and DTG scans for the pristine PEO and un-sintered ES PTFE/PEO and (b) DSC analysis of the un-sintered and sintered PTFE/PEO fibrous membranes. 

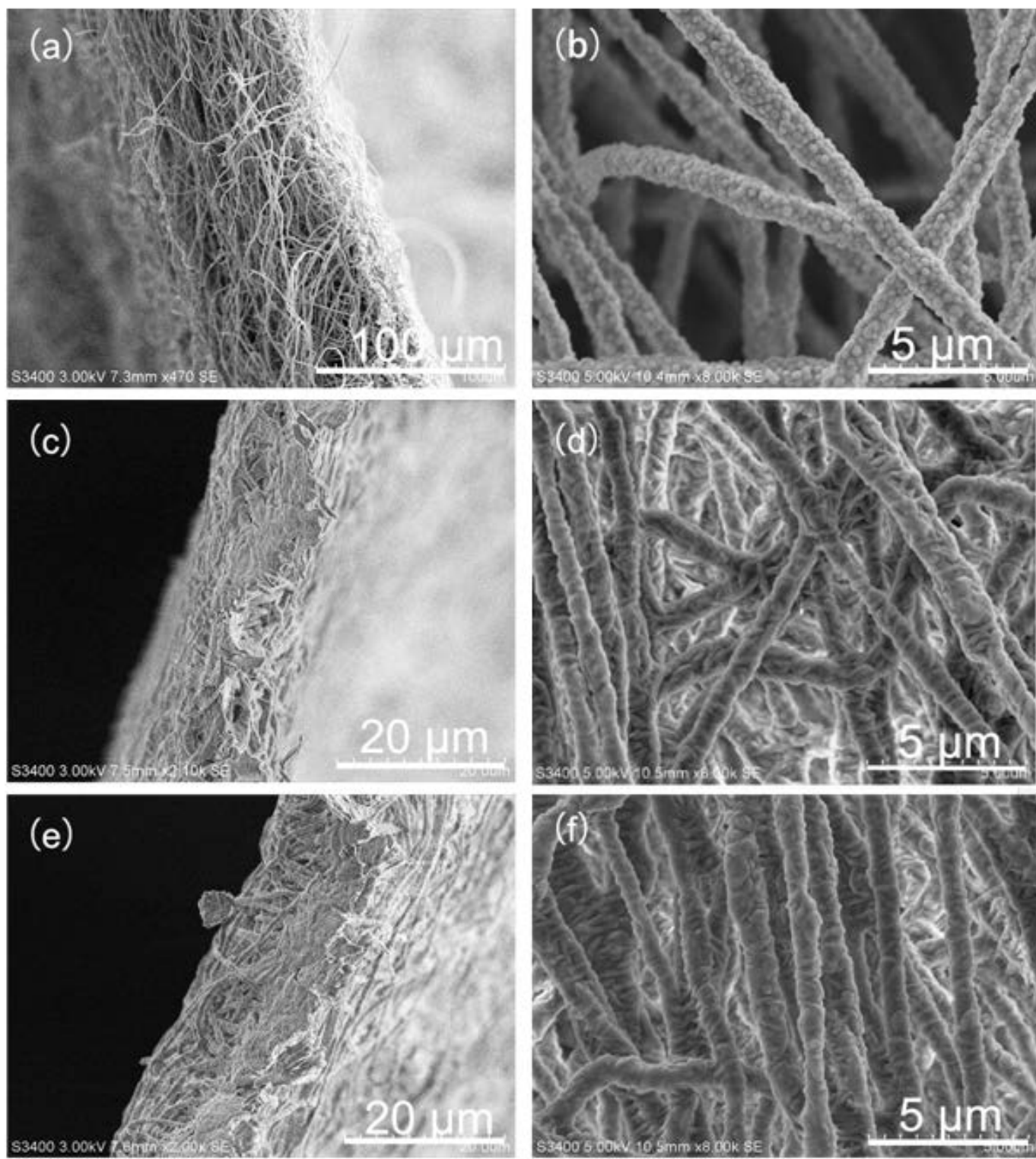

Figure 3. Fibre cross-section SEM images of a PEO/PTFE composite nanofibre membrane (a) before and after sintering process at (c) $350{ }^{\circ} \mathrm{C}$ and (e) $370{ }^{\circ} \mathrm{C}$, and surface morphologies of a pristine ES PTFE/PEO electrospun samples (b) before and after sintering process at (d) $350{ }^{\circ} \mathrm{C}$ and (f) $370{ }^{\circ} \mathrm{C}$, respectively. 

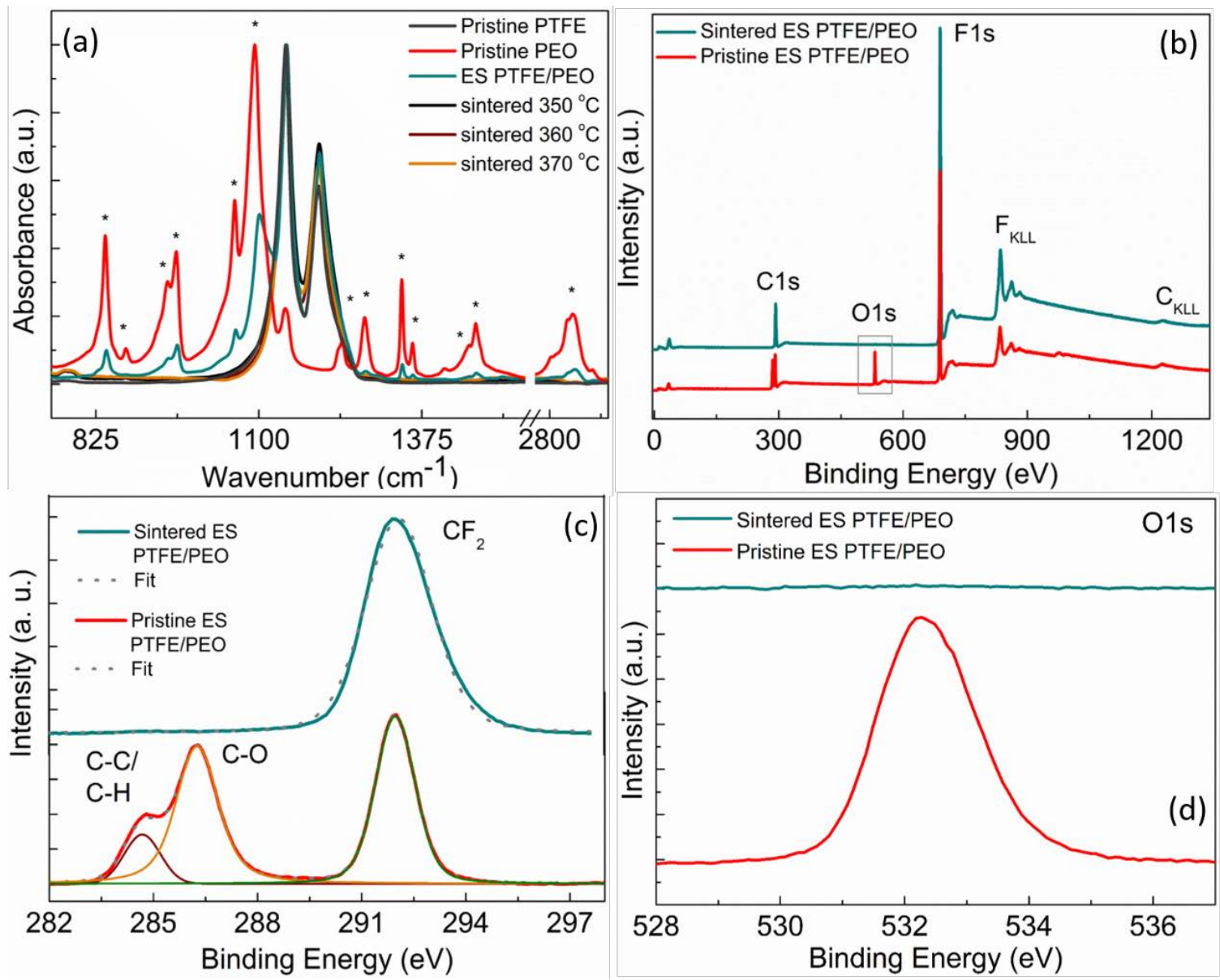

Figure 4. (a) FTIR absorption spectrum of samples including pristine PEO, PTFE and ES PTFE/PEO, before and after sintering at various temperatures, (b) wide energy survey spectrum (WESS) of an asdeposited pristine ES PTFE/PEO and sintered at $370{ }^{\circ} \mathrm{C}$, (c) core-level C1s and (d) O1s spectra showing the removal of oxygen species associated with the PEO after the sintering process. 

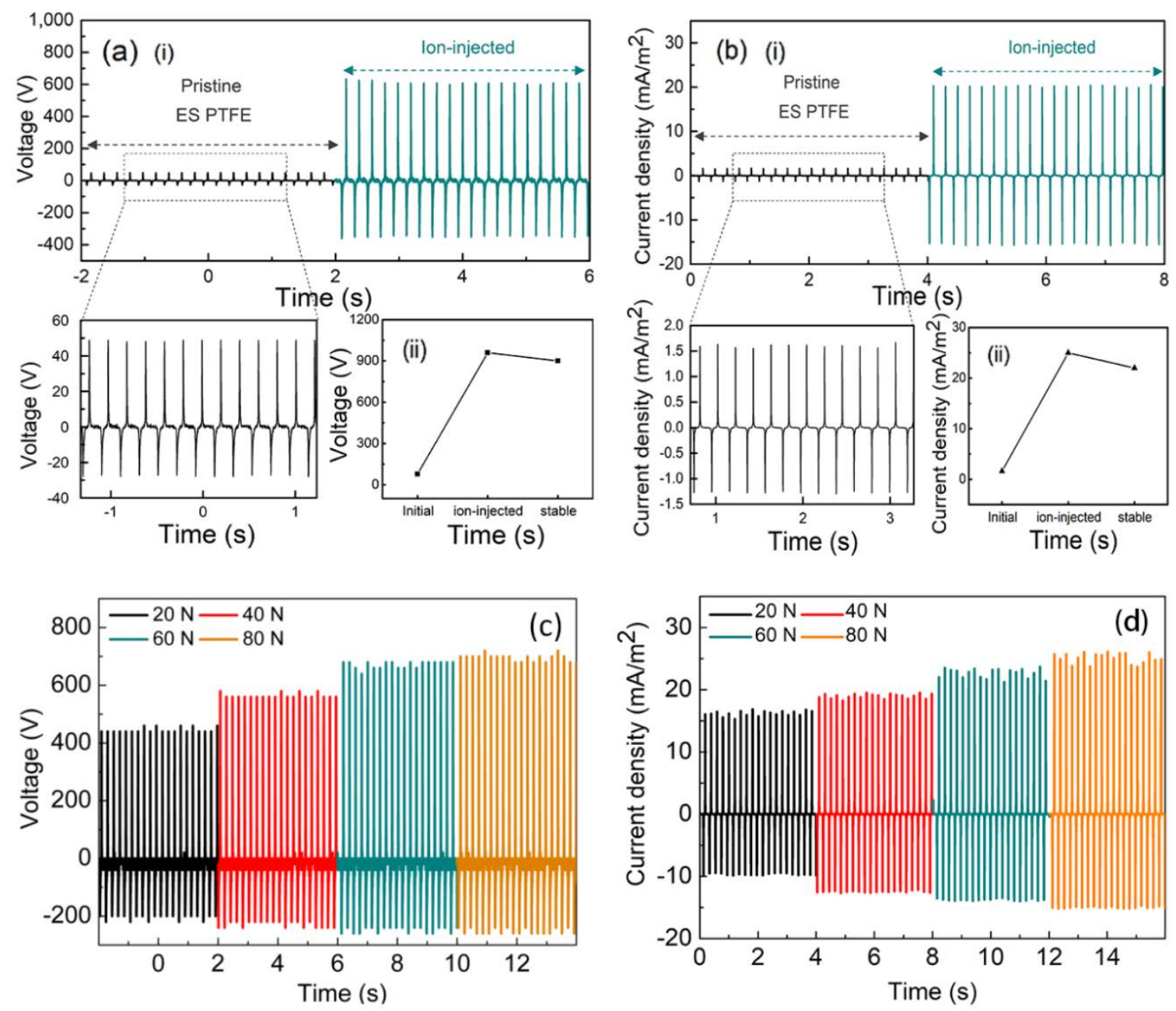

Figure 5. Electrical measurement results of the sintered ES PTFE/PA6 TENG, before and after ioninjection. The (a-i) open circuit voltage $\left(\mathrm{V}_{\mathrm{oc}}\right)$ and (b-i) short-circuit current density $\left(\mathrm{J}_{\mathrm{sc}}\right)$ measurements were carried out at an impact force of $50 \mathrm{~N}$, a frequency of $5 \mathrm{~Hz}$ and a spacer distance of $5 \mathrm{~mm}$ for the TENGs. Further measurements of $\mathrm{V}_{\text {ос }}$ and current density as a function of impact force on the ioninjected ES PTFE/PA6 TENG are shown in (c) and (d) respectively. The TENG had received one ioninjection process. The insets (ii) in (a) and (b) show the stability of open voltage and current density after the ion-injection step. 


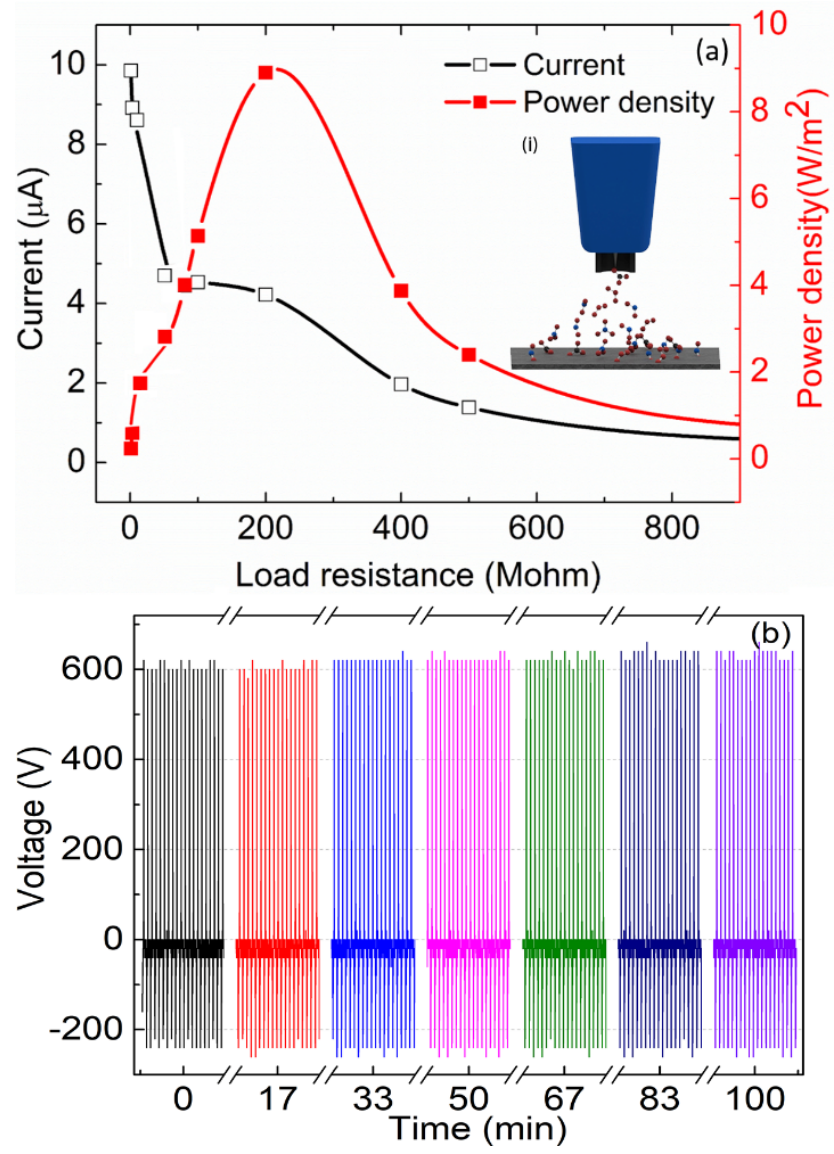

Figure 6. (a) Power density vs. load resistance curve, showing a maximum output power of $\sim 9 \mathrm{Wm}^{-2}$ at a load resistance of $200 \mathrm{M} \Omega$; the inset (i) is the schematic of the ion-injection process, (b) stability of the ion-injected surface charge on the ES PTFE surface as measured via the operation of ioninjected ES PTFE/PA6 TENG for 30,000 energy generation cycles at a working frequency of $5 \mathrm{~Hz}$. 

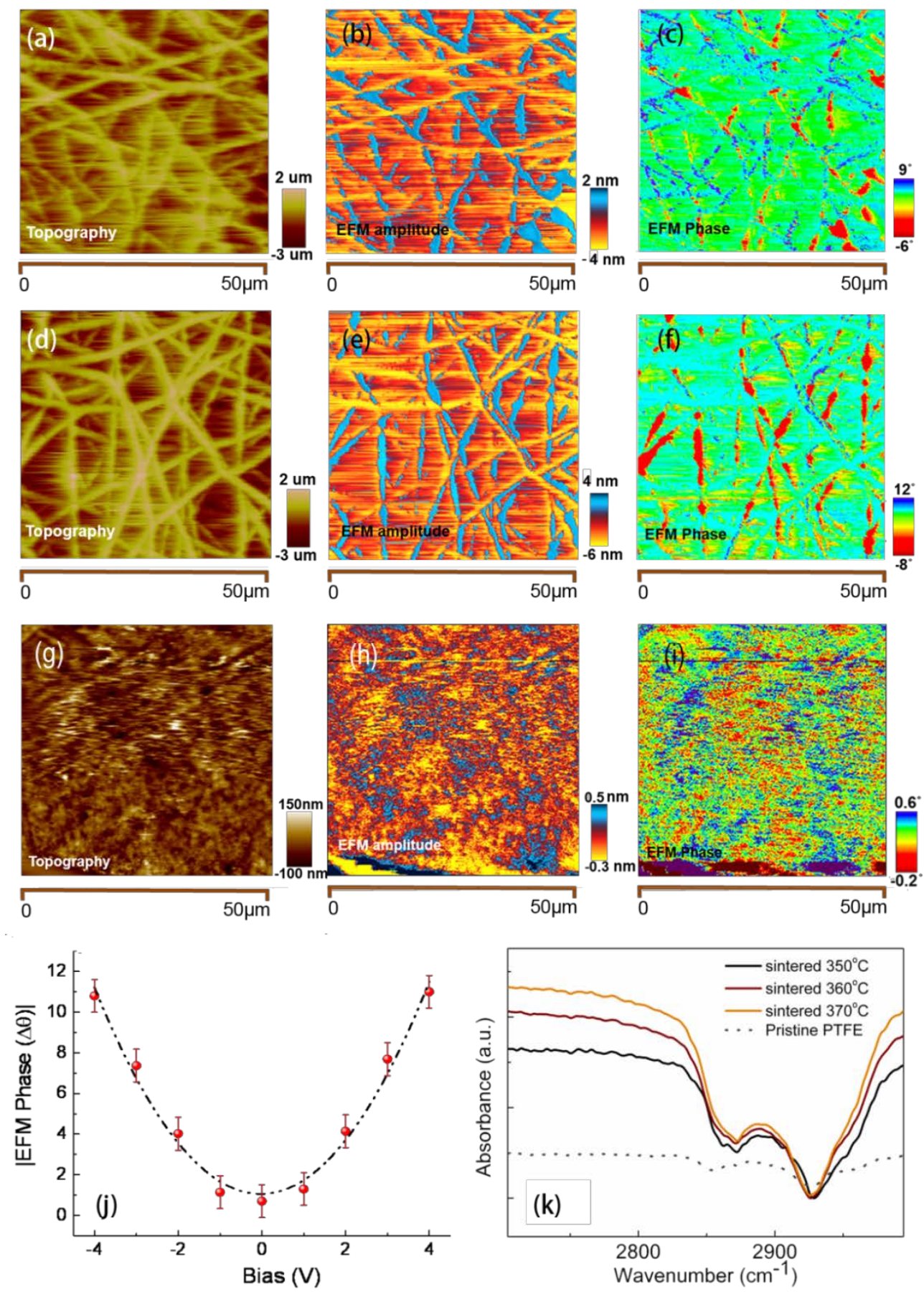

Figure 7. (a) Surface topography, (b) EFM amplitude and (c) EFM phase for the uncharged sintered ES PTFE nanofibers, with the corresponding images (d-f) shown after the ion-injection process. The EFM corresponding results of the commercial PTFE film are shown in the images (g-i). The increase in the EFM amplitude and phase difference can be clearly observed after the charge injection process and is indicative of the successful deposition of static negative charges on the surface. (j) EFM phase signal as a function of the probe-sample voltage at a scanning height of $200 \mathrm{~nm}$. (k) The increase in the intensity of C-H stretching band of FTIR spectrum in the post-sintering sample is indicative of the transfer of the positive charge from the PEO to PTFE. 


\section{TOC Graphic}
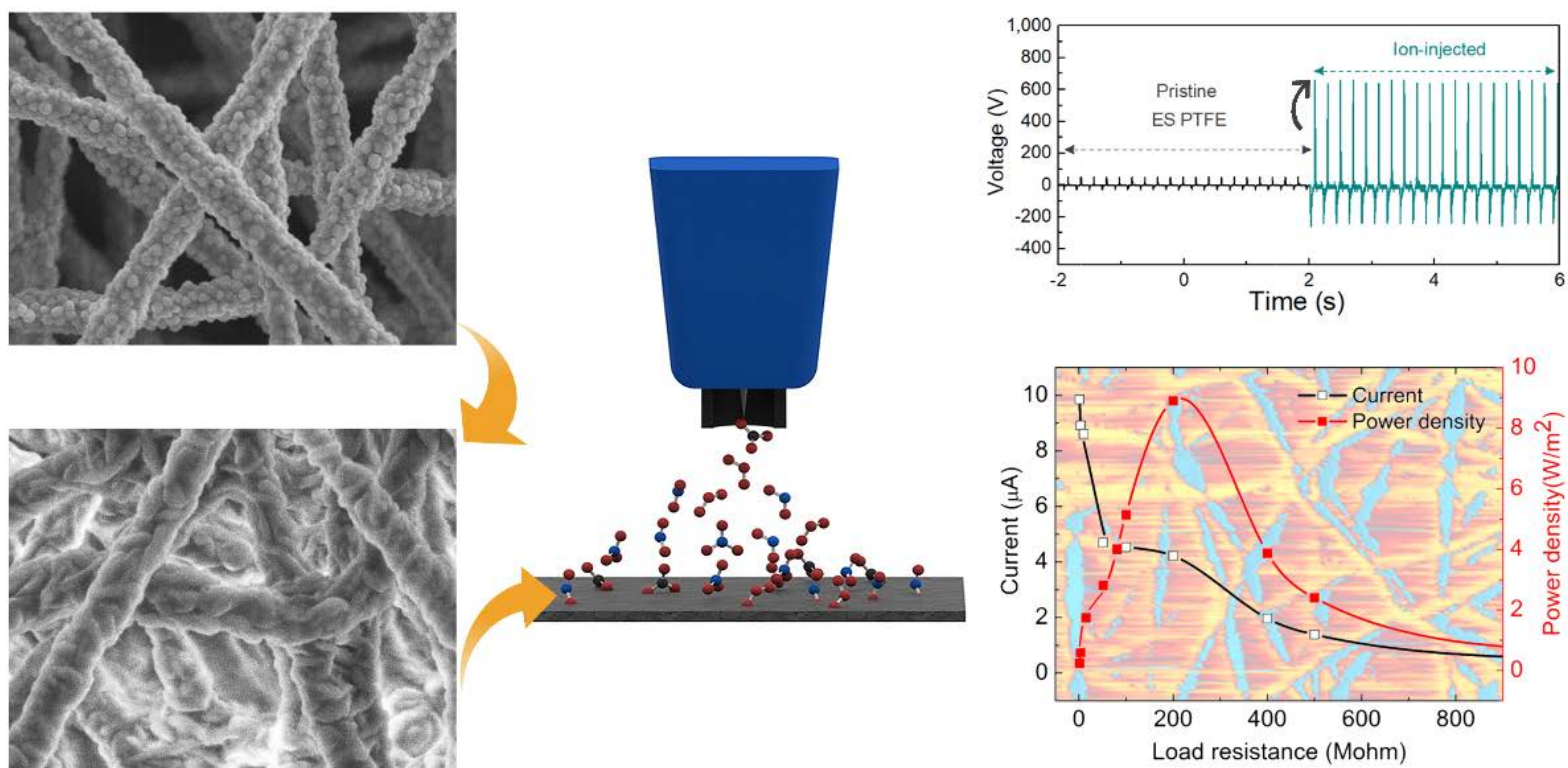